\title{
Integración didáctica de "apps" relacionadas con la producción animal en la docencia universitaria del Grado de Ingeniería Agroalimentaria y Agroambiental
}

\section{García M.L.", Agea I., y Argente M.J.}

Departamento de Tecnología Agroalimentaria. Universidad Miguel Hernández de Elche. Ctra. Beniel km 3.203312. Orihuela Alicante. España.

*email: mariluz.garcia@umh.es

\begin{abstract}
The objective of this work is the didactic integration of apps that are used in the management of livestock farms in the Degree in Agro-Food and Agro-Environmental Engineering. The academic results indicated that the scores improved by 1.67 points when the apps were included in the teaching. In addition, a questionnaire was carried out for the student, resulting that more than $66 \%$ of the students answered "Totally agree" to the sentence "The proposed task has made it easier for me to learn the subject". This was also the response for 33\% of the students to the sentences "The use of apps has helped me understand the subject" and "I would recommend the use of apps in other subjects of the subject". The answer "Neither agree nor disagree" was the answer selected by $66 \%$ of the students for the sentence "The use of apps has brought me closer to that of the livestock sector". The conclusions of this work are that the introduction of apps in teaching improves the academic performance of the student as they facilitate learning. It would be advisable to use this methodology to other subject.
\end{abstract}

Keywords: animal production, apps, teaching

\section{Resumen}

El objetivo de este trabajo es la integración didáctica de apps que se utilizan en la gestión de explotaciones ganaderas en el Grado de Ingeniería Agroalimentaria y Agroambiental. Los resultados académicos indicaron que las calificaciones mejoraron en 1.67 puntos cuando las apps fueron incluidas en la docencia. Además se realizó un cuestionario al estudiantado resultando que más del $66 \%$ del estudiantado respondió "Totalmente de acuerdo" a la sentencia "La tarea propuesta me ha facilitado el aprendizaje del tema". Ésta fue también la respuesta para el 33\% del estudiantado a las sentencias "La utilización de las apps me ha ayudado a comprender el tema" y "Recomendaría el uso de apps en otros temas de la asignatura". La respuesta "Ni de acuerdo ni en desacuerdo" fue la respuesta seleccionada por el 66\% del estudiantado para la sentencia "La utilización de las apps me ha acercado a la realidad del sector ganadero". Las conclusiones de este trabajo son que la incorporación de apps en la docencia mejora el rendimiento académico del estudiantado pues facilitan el aprendizaje. Sería aconsejable la utilización de esta metodología en otros temas de la asignatura.

Palabras clave: apps, docencia, producción animal 


\section{Introducción}

IAB (2019) señala que los usuarios de teléfonos móviles o smartphones utilizan 31 aplicaciones o apps de media en sus teléfonos, ascendiendo a 33 en los usuarios con una franja de edad de 18 a 24 años. La principal actividad realizada con los teléfonos móviles es social, como chatear o enviar mensajes privados y/o públicos a los contactos (65\%). El 28\% de la actividad se corresponde con fines profesionales o de estudio. Estos datos indican que es necesario considerar la integración de las nuevas tecnologías, y concretamente las apps, en el aula para la adquisición de conocimientos.

La ganadería digital se ha convertido en tendencia durante los últimos años. Se han desarrollado interesantes herramientas digitales, como apps, para facilitar la gestión del ganado en diferentes aspectos: gestión de la reproducción y de la condición corporal, monitorización de los animales en tiempo real, calendario de vacunación digitales, etc. (de Opazo, 2020). Estas apps son utilizadas cada vez con más asiduidad en la ganadería, por lo que es muy conveniente su explicación en las aulas para ampliar la formación de los futuros graduados.

Nuestra hipótesis es que el creciente uso de apps por parte de los universitarios podría ser aprovechado para la adquisición de conocimientos a través del uso de aplicaciones específicas que se utilizan usualmente en la ganadería. Por tanto, el objetivo de este trabajo es conocer el efecto de la integración en la docencia universitaria de apps comúnmente utilizadas por el sector ganadero.

\section{Desarrollo de la innovación}

La integración didáctica de las apps se realizó en la asignatura de Fundamentos de la Producción Animal que se imparte en el segundo semestre del tercer curso de la tecnología específica Mecanización y Construcciones Rurales del Grado en Ingeniería Agroalimentaria y Agroambiental en la Escuela Politécnica Superior de Orihuela de la Universidad Miguel Hernández de Elche.

Una de las competencias específicas de esta asignatura es "Capacidad para conocer, comprender y utilizar los principios de nutrición, higiene, sistemas de producción animal, biotecnología y mejora animal y productos animales”. Para su adquisición se incluyen los siguientes objetivos:

- Conocer la fisiología del crecimiento y de la reproducción de las principales especies ganaderas.

- Determinar los principales factores que afectan a la productividad de los animales.

- Realizar las principales actividades reproductivas de una explotación ganadera.

- Conocer el ciclo productivo de las principales especies ganaderas.

Para adquirir esta competencia se realizó una tarea sobre la condición corporal en las especies ganaderas de interés productivo, con las siguientes actividades:

- Una clase invertida dedicada a conocer y afianzar el concepto de condición corporal, las diferentes metodologías de medición, y la evolución de la condición corporal a lo largo de la vida productiva de los animales.

- Una clase práctica de medición de la condición corporal con diferentes metodologías en las granjas docentes de la Universidad Miguel Hernández de Elche.

En el curso actual, esta tarea fue ampliada incluyendo las siguientes actividades:

- Búsqueda de apps gratuitas en clase presencial que midan la condición corporal y su utilización con un ejemplo teórico. 
- Clase práctica que incluye la utilización de las apps en las granjas docentes de la Universidad Miguel Hernández de Elche.

Se evaluó la adquisición de las competencias mediante una prueba escrita. Las condiciones de entrenamiento para dicha prueba en ambos grupos de estudiantes (con y sin utilización de apps) fueron equivalentes.

Para conocer el efecto de la integración didáctica en el resultado del aprendizaje del estudiantado se valoraron las calificaciones obtenidas de la misma prueba escrita en ambos grupos. El análisis estadístico se realizó con metodología bayesiana. Se utilizó el programa Rabbit desarrollado por el Instituto de Ciencia Animal y Tecnología de la Universidad Politécnica de Valencia.

Para conocer el grado de aceptación por parte del estudiantado de esta actividad y su utilidad educativa se realizó una encuesta con las siguientes cuestiones:

1. La tarea propuesta me ha facilitado el aprendizaje del tema.

2. La utilización de las apps me ha ayudado a comprender el tema.

3. La utilización de las apps me ha acercado a la realidad del sector ganadero.

4. Recomendaría el uso de apps en otros temas de la asignatura.

Las respuestas se clasificaron en seis niveles:

a. Totalmente en desacuerdo

b. En desacuerdo

c. Ni de acuerdo ni en desacuerdo.

d. De acuerdo.

e. Totalmente de acuerdo

f. $\mathrm{NS} / \mathrm{NC}$

\section{Resultados}

La Tabla 1 muestra los parámetros de la distribución marginal posterior de la diferencia entre las calificaciones del grupo con apps y del grupo sin apps. La metodología Bayesiana nos permite hacer inferencia sobre las distribuciones marginales posteriores obtenidas y calcular con ellas las medianas de las calificaciones de cada grupo, así como la diferencia entre ellas (D), el HPD $95 \%$ y la probabilidad de que esta diferencia sea mayor de 0 . En este sentido, consideramos que si la probabilidad (P) de la diferencia entre las calificaciones de los grupos |D| es mayor de 0.90 hay suficiente evidencia para afirmar que las calificaciones entre los grupos difieren. La calificación del grupo con apps fue de 7.38 mientras que la del grupo sin apps fue de 5.71. Esto representa una diferencia de 1.67 puntos, con un $\mathrm{HPD}_{95 \%}$ que no contiene al cero $(0.15 ; 3.02)$ y siendo la probabilidad de que la diferencia sea mayor de 0 igual a 0.99 .

Los resultados indican que la metodología utilizada para el aprendizaje ha sido exitosa, pues el resultado de la evaluación de los conocimientos ha mejorado. 
Integración didáctica de "apps" relacionadas con la producción animal en la docencia universitaria del Grado de Ingeniería Agroalimentaria y Agroambiental

Tabla 1. Parámetros de la distribución marginal posterior de la diferencia entre las calificaciones del grupo con apps y sin apps para la calificación

\begin{tabular}{|c|c|c|c|c|c|}
\hline & Con $\operatorname{apps}(n=6)$ & Sin $\operatorname{apps}(\mathrm{n}=12)$ & D & HPD $_{95 \%}$ & $\mathbf{P}$ \\
\hline Calificación & 7.38 & 5.71 & 1.67 & $0.15 ; 3.02$ & 0.99 \\
\hline
\end{tabular}

D: mediana de la diferencia entre el grupo con apps y sin apps. HPD $95 \%$ : Región de alta densidad posterior al 95\%. P: Prob D>0.

Los resultados de la encuesta realizada al estudiantado sobre la actividad se muestran en la Figura 1. Destaca que ninguna de las sentencias del cuestionario fue contestada con NS/NC, "Totalmente en desacuerdo" ni "En desacuerdo". Más del 80\% del estudiantado reconoció que la utilización de las apps ha facilitado el aprendizaje del tema propuesto. Los resultados obtenidos en las calificaciones corroboran esta apreciación positiva del estudiantado sobre la tarea utilizada para el aprendizaje. La compresión del tema se vio favorecida con la utilización de las apps para el 50\% del estudiantado. Sin embargo resultó indiferente para el resto. La experiencia docente de años anteriores indica que el tema elegido para la utilización de esta experiencia piloto no es uno de los más complicados para su comprensión. Sería, por tanto, conveniente aplicar esta metodología docente en los temas más complejos para el estudiantado.

Para el $66.7 \%$ del estudiantado le resultó indiferente la utilización de las apps como un medio para acercarlos a la realidad del sector ganadero. Este resultado podría ser mejorado mediante seminarios o exposiciones de técnicos que utilicen habitualmente estas aplicaciones informáticas para la gestión de las explotaciones ganaderas.

Respecto a si otras competencias de la asignatura serían susceptibles de ser adquiridas utilizando esta metodología de aprendizaje, más del $80 \%$ del estudiantado recomendaría que esta metodología de trabajo fuese utilizada en otros temas de la asignatura. Por tanto, es necesario el estudio de la incorporación de esta innovación docente a otros temas de la asignatura, principalmente, como se ha comentado anteriormente, en aquellos temas con más dificultad para su comprensión.

\section{Conclusiones}

Con los resultados de este trabajo podemos concluir que la incorporación de apps en la docencia ha mejorado el rendimiento académico de nuestro estudiantado pues facilita el aprendizaje del tema. Además, es recomendable que el profesorado amplíe el ámbito de acción de esta innovación docente a otros temas de la asignatura, principalmente en aquellos temas con más dificultad de comprensión. 
Recomendaría el uso de apps en otros temas de la asignatura

La utilizaciónde las apps me ha acercado a la realidad del.

La utilización de las apps me ha ayudado a comprender el.

La tarea propuesta me han facilitado el aprendizaje del tema

\section{..}

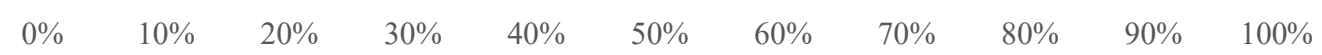

\begin{tabular}{|c|c|c|c|c|}
\hline & $\begin{array}{l}\text { La tarea propuesta me han } \\
\text { facilitado el aprendizaje del } \\
\text { tema }\end{array}$ & $\begin{array}{l}\text { La utilización de las apps me } \\
\text { ha ayudado a comprender el } \\
\text { tema }\end{array}$ & $\begin{array}{l}\text { La utilizaciónde las apps me } \\
\text { ha acercado a la realidad del } \\
\text { sector ganadero }\end{array}$ & $\begin{array}{l}\text { Recomendaría el uso de apps } \\
\text { en otros temas de la asignatura }\end{array}$ \\
\hline - NS/NC & 0,0 & 0,0 & 0,0 & 0,0 \\
\hline - Totalemente endesacuerdo & 0,0 & 0,0 & 0,0 & 0,0 \\
\hline En desacuerdo & 0,0 & 0,0 & 0,0 & 0,0 \\
\hline ni de acuerdo ni en desacuerdo & 16,7 & 50,0 & 66,7 & 16,7 \\
\hline De acuerdo & 16,7 & 16,7 & 33,3 & 50,0 \\
\hline Totalmente de acuerdo & 66,7 & 33,3 & 0,0 & 33,3 \\
\hline
\end{tabular}

$\square \mathrm{NS} / \mathrm{NC} \quad$ Totalemente endesacuerdo

En desacuerdo

- Ni de acuerdo ni en desacuerdo

- De acuerdo

$\square$ Totalmente de acuerdo

Fig. 1 Resultados de la encuesta realizada al estudiantado 
Integración didáctica de "apps" relacionadas con la producción animal en la docencia universitaria del Grado de Ingeniería Agroalimentaria y Agroambiental

\section{Agradecimientos}

Este trabajo ha sido financiado por la Universidad Miguel Hernández de Elche a través del Programa PIEUUMH 2019/20.

\section{Referencias}

DE OPAZO, J.G. Ganadería digital, herramientas y APPs para la granja del siglo XXI. https://lahuertadigital.es/ganaderia-digital-herramientas-y-apps-para-la-granja-del-siglo-Xxi/[Consulta: 15 de marzo de 2020]

IAB (2019). Estudio anual de redes sociales 2019. https://iabspain.es/estudio/estudio-anual-de-redes-sociales2019/[Consulta 15 de marzo 2020] 